\title{
Caractérisation agromorphologique des variétés de niébé cultivées au Bénin
}

\author{
A. A. GBAGUIDI ${ }^{1}$, P. ASSOGBA ${ }^{1}$, M. DANSI ${ }^{1}$, H. YEDOMONHAN $^{2}$ et A. DANSI ${ }^{1 *}$ \\ ${ }^{1}$ Laboratoire de Biotechnologies, Ressources Génétiques et Amélioration des Espèces \\ Animales et Végétales (BIORAVE), Faculté des Sciences et Techniques de Dassa, \\ Université Polytechnique d'Abomey, BP 14 Dassa, Bénin. \\ ${ }^{2}$ Laboratoire de Botanique et Ecologie Végétale (LaBEV), Université d'Abomey-Calavi (UAC), 01 BP 4521 \\ Cotonou, Bénin. \\ "Auteur correspondant, E-mail: adansi2001@gmail.com
}

\section{RESUME}

Le niébé (Vigna unguiculata (L.) Walp.) est une légumineuse très cultivée et consommée au Bénin. Sa diversité reste encore peu connue et l'utilisation des variétés traditionnelles disponibles à des fins de sélection exige une bonne connaissance de leurs caractéristiques agronomiques, technologiques et culinaires. Pour cette raison, 124 variétés traditionnelles collectées dans différentes localités ont été évaluées sur la base de 11 variables quantitatives et 12 variables qualitatives dans un dispositif de blocs complètement aléatoire (BCA) à trois répétitions. Parmi les caractères qualitatifs, la collection analysée fait montre d'une importante variabilité (42 morphotypes) pour le port de la tige, la pigmentation de la plante, la couleur des fleurs, la forme des folioles, la couleur des gousses, la taille, la couleur et la formes des graines. L'analyse en composante principale (ACP) des variables quantitatives a révélé 4 groupes au sein de la collection dont le groupe (G1) rassemble la plupart des variétés très performantes (cycle court, nombre de gousses élevé par plante, grains gros et lourds, rendements supérieurs à 2 tonnes à l'hectare) comme Catché godonou, Ewaoloy, Gbolékpomin, Kaki et Yanbodo qu'on peut utiliser dans les programmes de sélection. Une corrélation positive significative est observée entre le rendement par plante de niébé et le nombre de gousses par plant (Ngs), la longueur des gousses (LGs) et le poids de 100 graines (P100G). Ces données exploitables pour la sélection de variétés améliorées et la gestion des ressources génétiques du niébé doivent être encore complétées par la caractérisation moléculaire.

() 2015 International Formulae Group. All rights reserved.

Mots clés : Bénin, diversité, niébé, rendement, variabilité morphologique.

\section{INTRODUCTION}

Le niébé [Vigna unguiculata (L.) Walp.] constitue l'une des principales légumineuses cultivées et consommées dans les zones tropicales et subtropicales de l'Afrique, d'Asie, d'Europe et d'Amérique (Taffouo et al., 2008). C'est une espèce diploïde $(2 \mathrm{n}=2 \mathrm{x}=22)$ de la famille des Fabaceae qui serait originaire d'Afrique (Coulibaly et al., 2002 ; Kuruma et al., 2008). La production annuelle mondiale varie entre 3 et 5,5 millions de tonnes de graines sèches (FAOSTAT, 2010; Shanko et al., 2014 ; Makanur et al., 2013) dont plus de 64\% sont produits en Afrique (Nkouannessi, 2005). La superficie annuelle cultivée dans le monde s'élève à plus de 11,8 millions d'hectares dont 10,7 millions d'hectares en Afrique de l'Ouest, la plus grande zone de production et de consommation du niébé dans le monde (Ouali-N'goran et al., 2014). 
En raison de sa forte teneur en protéines (19 à 25\%), en carbohydrates et en éléments minéraux, le niébé joue un rôle important dans l'alimentation humaine et dans la lutte contre la malnutrition (Stoilova et Pereira, 2013). Les graines, les gousses fraîches et les feuilles sont consommées dans beaucoup de pays africains; les feuilles sont également utilisées dans l'alimentation du bétail (Abebe et al., 2005). Grâce à sa capacité de fixation symbiotique de l'azote atmosphérique, l'insertion du niébé dans les rotations culturales permet de combler les besoins en engrais azoté des cultures subséquentes (Carsky et al., 2002; Tarawali et al., 2002; Sanginga et al., 2003).

Au Bénin, le niébé est cultivé sur toute l'étendue du territoire principalement pour ses graines comestibles (Gbaguidi et al., 2013) et il occupe $7 \%$ des superficies emblavées pour les cultures annuelles avec une production de 11224 tonnes (MAEP, 2012; Abadassi, 2014). Malheureusement, sa production est confrontée à plusieurs contraintes d'ordre biotiques et abiotiques dont les attaques des graines par des insectes au champ et au cours du stockage (Niba, 2011; Houinsou et al., 2014). L'évaluation avec les producteurs des variétés cultivées a révélé l'existence de variétés performantes pouvant répondre aux nouvelles conditions liées aux changements climatiques (Gbaguidi et al., 2013). A l'exception de quelques variétés étudiées par Zannou et al. (2004) et Abadassi (2014), la diversité variétale du niébé au Bénin reste peu connue. Pour des programmes de sélection et d'amélioration variétale, la connaissance de la diversité des variétés traditionnelles et leur évaluation sont nécessaires (Hedge et Mishra, 2009; Doumbia et al., 2013).

Nous présentons dans cet article les résultats d'une étude agromorphologique qui a pour objectifs d'analyser la variabilité morphologique au sein de la collection nationale, d'identifier au sein de cette collection des variétés élites exploitables dans les programmes de vulgarisation et de sélections et d'analyser les relations entre les variables quantitatives qui influencent le rendement.

\section{MATERIEL ET METHODES}

Le matériel végétal est constitué de cent-vingt-quatre (124) variétés traditionnelles différemment nommées qui ont été collectées dans 84 villages à travers toutes les zones agroécologiques du Bénin. L'étude a été conduite sur le site expérimental de la Faculté des Sciences et Techniques de Dassa, dans la zone soudano-guinéenne caractérisée par deux saisons pluvieuses et deux saisons sèches avec une pluviométrie moyenne de $1200 \mathrm{~mm} / \mathrm{an}$ (Akoègninou et al., 2006).

Les graines ont été semées en culture pure sur une jachère avec un espacement de $0,75 \mathrm{~m}$ sur des billons espacés de $0,75 \mathrm{~m}$ dans un dispositif expérimental de blocs complètement aléatoires avec trois répétitions. Douze (12) variables qualitatives et onze (11) variables quantitatives (Tableau 1) ont été considérées pour la caractérisation agromorphologique. Les données ont été prises suivant les recommandations inscrites dans les descripteurs du niébé (IBPGR, 1983) et d'après Cobbinah et al. (2011) (Tableau 1).

\section{Analyses statistiques}

Les données ont été analysées par la statistique descriptive (moyenne, pourcentage, variance, etc.) et les résultats sont présentés sous forme de tableaux et de figures. Les corrélations entre les variables quantitatives ont été estimées par le coefficient de corrélation de Pearson avec le logiciel minitab (Minitab version 14, Minitab Inc., State College, PA, USA). Pour analyser les relations entre les variétés étudiées sur la base des variables qualitatives, celles-ci ont été considérées, selon Kombo et al. (2012), comme des individus et les traits morphologiques qualitatifs comme des variables et codées par des chiffres (Tableau 1). La base de données obtenue est utilisée pour construire une matrice de similarité et un dendrogramme avec la méthode UPGMA (Unweighted Pair-Group Method with Arithmetic Average) et le logiciel NTSYS-pc 2.2 (Sneath et Sokal, 1973 ; Swofford et Olsen, 1990 ; Rohlf, 2009). Une analyse en composante principale (ACP) a été aussi faite selon Adjatin et al. (2013) avec Minitab (Minitab la version 14, Minitab Inc., State College, PA, USA) en utilisant uniquement 
les données quantitatives pour identifier les variétés à haute performance.

\section{RESULTATS}

\section{Analyse des variables qualitatives}

L'étude a montré que la majorité des variétés traditionnelles $(65,32 \%)$ présentent des plants faiblement pigmentés, $33,87 \%$ sont modérément pigmentés et seulement $0,81 \%$ sont très pigmentées (Figure 1a); les folioles sont de couleurs vert foncées $(71,77 \%$ des variétés) ou vert claires $(28,23 \%$ des variétés) (Figure 1b); 45,16\% des variétés de niébé présentent un port rampant, 33,06\% ont un port semi érigé et $21,78 \%$ présentent un port érigé (Figure 1c). Les fleurs (Figure 1d) ont principalement la couleur blanche $(58,87 \%)$ et violette $(41,13 \%)$. Dans la collection analysée, $71,77 \%$ de variétés ont des folioles globulaires, $27,43 \%$ des variétés ont des feuilles sub-globulaires et seulement $0,80 \%$ ont des feuilles lancéolées (Figure 1e). La majorité des variétés $(90,32 \%)$ ont des gousses de couleur crème, $5,64 \%$ ont des gousses violettes, seulement 3,22 et $0,82 \%$ ont respectivement des gousses de couleur noires et jaunâtre (Figure 1f). Les caractéristiques des graines (couleur, taille, forme, aspects et autres traits particuliers) sont représentées sous forme de fréquence par les histogrammes des Figures 1g, 1h, 1i, $1 \mathrm{j}$ et $1 \mathrm{k}$.

Le dendrogramme issu de la combinaison des variables qualitatives classe les 124 accessions en 42 unités morphologiques (Figure 2). A $62 \%$ de similarité, les 42 unités sont classées en 6 classes : La classe 1 est constituée des unités (M1 à M16) caractérisées par des folioles globulaires (Ffo1), des gousses de couleur crème (CGs1) avec des graines à tégument blancs (CGr1) et rugueux (AGr1). La variété Assoban'dé qui forme l'unité M17 constitue la classe 2 et est caractérisée par une forte pigmentation (Pgt3) de la plante, un port érigé (PrT3) avec des feuilles sub-globulaire (Ffe3), des gousses de couleur violette (CGs2) et des graines de petites tailles (TGr1). La classe 3 est formée des unités M18 à M21 constituées des variétés à pigmentation moyenne (Pgt 2), à port semi érigé (PrT2), avec des folioles de forme globulaire (Ffo1) et de couleur vert claire (Cfo1); la classe 4 est constituée des unités M22 à M26 avec une faible pigmentation (Pgt1) des variétés, à foliole de couleur vert claire (Cfol), à fleurs violettes et des graines à yeux noirs (Coi1), de petites tailles (TGr1) avec la partie basale colorée en rouge (TpG4); les unités M27, M28 et M29 constituent la classe 5 qui regroupe des variétés à tige faiblement pigmentée (TpG1) avec des folioles de couleur vert claire (Cfo1), de forme sub-globulaire (Ffe3) avec des graines de couleur rose. Les variétés de la classe 6 (M30 à M42) ont des folioles de couleur vert claire (Cfo1), des fleurs violettes (CoF2) avec des feuilles de forme subglobulaire.

\section{Analyse des variables quantitatives}

Les variables quantitatives étudiées au niveau des 124 accessions ont révélé des écarts importants entre les valeurs minimales et maximales avec des coefficients de variation qui vont de $24,20 \%$ (Temps de maturation) à $85,75 \%$ (longueur des gousses). On note des variations significatives $(\mathrm{Cv}>20 \%)$ pour toutes les variables quantitatives étudiées (Tableau 2).

Les dates de floraison varient entre 31 et 66 jours après semis (JAS). 26,61\% des variétés fleurissent entre 31 et 39 jours après semis (JAS). C'est le cas de: Sakaoga (31JAS), Kpodjiguèguè (35 JAS) et de Narétchagué (39 JAS); 15,32\% des variétés fleurissent entre 44 et $47 \mathrm{JAS} ; 12,91 \%$ des variétés fleurissent entre 56 et 59 JAS ; $45,16 \%$ des variétés fleurissent entre 60 et 66 JAS (Figure 3), c'est le cas de: Tawa (60 JAS), Atchawé (61 JAS) et de Sounansoé (66 JAS).

La majorité des variétés $(54,04 \%)$ ont des cycles supérieurs ou égal à 3 mois (Figure 4) ; il s'agit principalement de Ewaoloy (93 JAS), Tinyrma (92 JAS), Tola (90 JAS) ; $12,9 \%$ des variétés sont semi précoces avec des cycles variant entre 75 et 85 jours après semis. Les variétés très précoces d'environ 2 mois font 33,06\%. Par exemple, Assoban'dé, Mahunan et Kpodjiguèguè murissent respectivement à 52, 60 et 65 jours après semis.

Les variétés ayant les poids de 100 graines les plus élevés sont Eva oloy, Doungoudiibi et Ayicounvovo avec 
respectivement $23,75 \mathrm{~g}, 22,98 \mathrm{~g}$ et $21,67 \mathrm{~g}$ alors que les variétés Yandaha, yanguicorona et Etounpea ont présenté les poids les plus faibles avec respectivement $4 \mathrm{~g}, 4,05 \mathrm{~g}$ et $4,27 \mathrm{~g}$. Quant au rendement en $\mathrm{Kg} / \mathrm{ha}$, les variétés Ewa oloy, Kaki et Catche godonou ont les rendements les plus élevés avec respectivement $2696,5 \mathrm{Kg} / \mathrm{ha}, 2684,2 \mathrm{Kg} / \mathrm{ha}$ et $2617,3 \mathrm{Kg} / \mathrm{ha}$. Les accessions comme Ayankoko, Tinperga et Katoupiene ont les rendements les plus faibles avec moins de 15 $\mathrm{Kg} / \mathrm{ha}$.

Des corrélations positives et hautement significatives (Tableau 3) ont été observées entre le temps de floraison et le temps de maturation $(\mathrm{r}=0,77 ; \mathrm{p}=0,0001)$, entre le nombre de gousses et le poids de 100 graines $(r=1,00 ; p=0,0001)$; entre la longueur des gousses et le rendement en $\mathrm{Kg} / \mathrm{ha}(\mathrm{r}=0,68$; $\mathrm{p}=0,0001)$. Des corrélations négatives, hautement significatives ont été observées entre le temps de maturation et le nombre de gousses $(\mathrm{r}=-0,74 ; \mathrm{p}=0,0001)$ d'une part et entre le temps d'émergence et le poids de 100 graines d'autre part $(\mathrm{r}=-0,31 ; \mathrm{p}=0,001)$ (Tableau 3).

\section{Structuration de la diversité à partir des variables quantitatives}

Les trois premiers axes de l'analyse en composantes principales (ACP) expliquent respectivement $36,72 \% ; 13,49 \%$ et $9,95 \%$ de la variabilité, soit $60,16 \%$ de la variabilité totale. Les deux premières composantes qui absorbent $50,21 \%$ de la variance sont retenues pour analyser la variabilité agromorphologique des variétés. L'axe 1 exprime le développement végétatif et phénologique. Le temps de floraison (Tfl) et le temps de maturation (Tma) sont corrélés positivement à cet axe 1 tandis que le nombre de gousse (NGs), la longueur des gousses (LGs), le poids de 100 graines (Pd100G) et le rendement en graines (Rend) en $\mathrm{Kg} / \mathrm{ha}$ sont corrélés négativement à l'axe. L'axe 2 définit la longueur et la largeur des graines (Tableau 4). Les caractères qui contribuent à la formation de cet axe 2 sont négativement corrélés à l'axe. La projection des 124 individus dans le plan factoriel défini par les axes 1 et 2 (Figure 5) met en évidence quatre groupes. Le groupe 1 (G1) est constitué des variétés à rendement élevé et à graines de petite et moyenne taille, tardives ( $>90 \mathrm{JAS}$ ) ou très précoces $(<60 \mathrm{JAS})$. Les variétés Ewa oloy (N120) et Kaki (N113) font partie de ce groupe avec un rendement supérieur à 2000 $\mathrm{Kg}$ par hectare. Dans ce groupe, les variétés N81 (Narétchagué), N84 (Natindji), N89 (Abobo), N102 (Yanbodo) sont très précoces (durée du cycle $<60$ jours après semis) en plus de la forte productivité. Le groupe 2 (G2) est caractérisé par des variétés à rendement moyen ou faible et à cycle court de 60 jours après semi ou semi tardif d'environ 80 JAS. Les groupes 3 et 4 sont constitués d'individus à faible rendement et à cycle tardif (durée du cycle >90 JAS). Le groupe 3 (G3) est formé de variétés dont les graines sont de grosses ou moyennes tailles alors que le groupe 4 (G4) est formé des graines de petites ou de tailles moyennes (Figure 3).

\section{Sélection des variétés élites des morphotypes}

La caractérisation agromorphologique a permis d'identifier des variétés hors types au sein des morphotypes constitués. Par exemple, les variétés N12 (Azayu Wlétchivé) et N34 (Baguédou) qui étaient dans le morphotype 1 à cause de la couleur des graines (Figure 6) se distinguent par le port érigé de la variété N12 (Figure 6a1) et le port rampant de N34. N12 avec un rendement de $929,7 \mathrm{Kg} / \mathrm{ha}$ est la variété élite du morphotype. Les variétés N89 (Abobo) et N91 (Kpodjiguèguè) se distinguent des autres variétés de leur morphotype. La variété N89 est caractérisée par un port érigé, des feuilles lancéolées, des gousses de couleur violette et des graines de couleur rougeâtre (Figures 6b1, 6b3 et 6b4) différente de ce qui a été semé avec un rendement de $1014 \mathrm{Kg} / \mathrm{ha}$. La variété N91 très précoce est caractérisée par un port semi rampant, des feuilles larges (Figure 6c) avec un rendement de 1264,5 $\mathrm{Kg} / \mathrm{ha}$. Cette dernière a été identifiée comme élite dans le morphotype.

$\mathrm{Au}$ total, vingt-six variétés ont été identifiées comme élites au niveau des morphotypes (Tableau 5). Ces variétés ont été sélectionnées sur la base des paramètres quantitatifs tels que la précocité, la longueur des gousses, le nombre de gousses par plant, le poids de 100 graines et le rendement des graines en $\mathrm{Kg} / \mathrm{ha}$. 
Tableau 1: Variables utilisées pour la caractérisation agromorphologique (IBPGR, 1983).

\begin{tabular}{|c|c|c|c|}
\hline $\mathrm{N}^{\circ}$ & Variables quantitatives & Code & Description et collecte de données \\
\hline 1 & Temps d'émergence & Tem & Date 50\% émergence \\
\hline 2 & Temps de floraison & Tfl & Date $50 \%$ floraison \\
\hline 3 & Temps de maturation & Tma & Date de la première grande récolte \\
\hline 4 & Nombre de branches & $\mathrm{NBr}$ & $\begin{array}{l}\text { Compter le nombre de branches par } \\
\text { plante sur les } 3 \text { répétitions }\end{array}$ \\
\hline 5 & Nombre de fleurs par pédoncule & NFp & $\begin{array}{l}\text { Compter le nombre de fleurs de } 3 \\
\text { pédoncules par plane sur les } 3 \\
\text { répétitions }\end{array}$ \\
\hline 6 & Nombre de gousses par plant & Ngs & $\begin{array}{l}\text { Compter le nombre de gousses par } \\
\text { plante à la fin des récoltes }\end{array}$ \\
\hline 7 & Longueur des gousses & LGs & $\begin{array}{c}\text { Mesure de la longueur de } 3 \text { gousses } \\
\text { saines séchées par plante sur les } 3 \\
\text { répétitions }\end{array}$ \\
\hline 8 & Longueur des graines & LGr & $\begin{array}{l}\text { Mesure de la longueur de } 3 \text { graines par } \\
\text { gousse, par plant et par accession }\end{array}$ \\
\hline 9 & Largeur des graines & $1 \mathrm{Gr}$ & $\begin{array}{l}\text { Mesure de la largeur de } 3 \text { graines par } \\
\text { gousse, par plant et par accession }\end{array}$ \\
\hline 10 & Poids de 100 graines & P100G & $\begin{array}{l}\text { Compter et peser } 100 \text { graines de chaque } \\
\text { accession sur les } 3 \text { répétitions }\end{array}$ \\
\hline \multirow[t]{2}{*}{11} & Rendement des graines par ha & Rend $\mathrm{Kg} / \mathrm{ha}$ & $\begin{array}{l}\text { Peser le poids des graines d'un plant } \\
\text { pour estimer le poids (Kg/ha) }\end{array}$ \\
\hline & Variables qualitatives & & \\
\hline 12 & Pigmentation de la tige & Pgt & 1) Faible, 2) Moyenne, 3) Forte \\
\hline 13 & Couleur de la foliole & Cfo & 1) Vert clair, 2) Vert foncé \\
\hline 14 & Port de la tige & PrT & 1) Rampant, 2) Semi érigé, 3) Erigé \\
\hline 15 & Couleur de la fleur & $\mathrm{CoF}$ & 1) Blanc, 2) Violet \\
\hline 16 & Forme des feuilles & Ffe & $\begin{array}{l}\text { 1) Globulaire, 2) Lancéolé, 3) Sub } \\
\text { globulaire }\end{array}$ \\
\hline 17 & Couleur gousse & CGs & 1) Crème, 2) Violet, 3) Noir, 4) Jaunâtre \\
\hline 18 & Couleur des graines & CGr & $\begin{array}{l}\text { 1) Blanc, 2) Rose, 3) Rouge, 4) Rose } \\
\text { clair, 5) Rose jaune, 6) Violet, 7) } \\
\text { Cendre, 8) Crème-Rose }\end{array}$ \\
\hline 19 & Couleur de l'œil & Coi & 1) Noir, 2) Rouge, 3) Rose, 4) Blanc \\
\hline 20 & Taille des graines & TGr & 1) Petite, 2) Moyenne, 3) grande \\
\hline 21 & Aspect des graines & AGr & 1) Rugueux, 2) Lisse \\
\hline 22 & Forme des graines & FGr & 1) Arrondi, 2) eu allongé, 3) Allongé \\
\hline 23 & Traits particuliers des graines & TpG & $\begin{array}{c}\text { 0) Aucun, 1) Tache noire à la base, 2) } \\
\text { base colorée en rouge, 3) Bande noire à } \\
\text { la base, 4) Petit point noir sur le } \\
\text { tégument, 5) Tache rose sur tout le } \\
\text { tégument, 6) Tache noire sur tout le sur } \\
\text { tout le tégument, 7) Tache brune sur le } \\
\text { tégument }\end{array}$ \\
\hline
\end{tabular}


Tableau 2: Analyse descriptive des variables quantitatives.

\begin{tabular}{lcccccc}
\hline Caractères & Moyenne & Minimum & Maximum & Variance & Cv & Ecart-type \\
\hline Tem & 3,8548 & 3,00 & 5,00 & 0,65 & 20,84 & 0,80 \\
TFl & 52,1694 & 31,00 & 66,00 & 147,04 & 23,24 & 12,12 \\
NBr & 8,46 & 5,00 & 12,33 & 3,05 & 20,64 & 1,74 \\
Tma & 77,12 & 52,00 & 93,00 & 348,29 & 24,20 & 18,66 \\
NFp & 5,46 & 3,00 & 9,00 & 1,65 & 23,54 & 1,28 \\
NGs & 25,51 & 5,00 & 70,66 & 353,21 & 73,66 & 18,79 \\
LGS & 16,01 & 10,83 & 22,00 & 4,34 & 85,75 & 2,08 \\
LGr & 0,41 & 0,10 & 0,70 & 0,01 & 23,77 & 0,09 \\
lGr & 0,26 & 0,09 & 0,41 & 0,00 & 25,53 & 0,06 \\
Pd100G & 8,73 & 4,00 & 23,75 & 39,15 & 71,65 & 6,25 \\
Ren Kg/ha & 969,1 & 11,37 & 2696,5 & 9876,41 & 68,09 & 202,17 \\
\hline
\end{tabular}

Tableau 3: Corrélations entre les variables quantitatives.

\begin{tabular}{|c|c|c|c|c|c|c|c|c|c|c|c|}
\hline & Tem & TFl & NBr & Tma & NFp & NGs & LGS & LGr & lGr & Pd100G & PGm2 \\
\hline Tem & 1,00 & & & & & & & & & & \\
\hline TFl & $\begin{array}{c}0,27 \\
0,003 \\
\end{array}$ & 1,00 & & & & & & & & & \\
\hline $\mathrm{NBr}$ & $\begin{array}{c}0,09 \\
0,324 \\
\end{array}$ & $\begin{array}{c}0,45 \\
0,000\end{array}$ & 1,00 & & & & & & & & \\
\hline Tma & $\begin{array}{c}0,38 \\
0,000 \\
\end{array}$ & $\begin{array}{c}0,77 \\
0,000 \\
\end{array}$ & $\begin{array}{c}0,51 \\
0,000 \\
\end{array}$ & 1,00 & & & & & & & \\
\hline NFp & $\begin{array}{l}-0,05 \\
0,546 \\
\end{array}$ & $\begin{array}{l}-0,32 \\
0,000 \\
\end{array}$ & $\begin{array}{l}-0,23 \\
0,010 \\
\end{array}$ & $\begin{array}{l}-0,24 \\
0,007 \\
\end{array}$ & 1,00 & & & & & & \\
\hline NGs & $\begin{array}{l}-0,31 \\
0,001 \\
\end{array}$ & $\begin{array}{l}-0,49 \\
0,000 \\
\end{array}$ & $\begin{array}{l}-0,44 \\
0,000 \\
\end{array}$ & $\begin{array}{l}-0,74 \\
0,000 \\
\end{array}$ & $\begin{array}{c}0,12 \\
0,182 \\
\end{array}$ & 1,00 & & & & & \\
\hline LGS & $\begin{array}{l}-0,04 \\
0,630 \\
\end{array}$ & $\begin{array}{l}-0,25 \\
0,006 \\
\end{array}$ & $\begin{array}{l}-0,31 \\
0,000 \\
\end{array}$ & $\begin{array}{l}-0,38 \\
0,000 \\
\end{array}$ & $\begin{array}{c}0,21 \\
0,020 \\
\end{array}$ & $\begin{array}{c}0,48 \\
0,000 \\
\end{array}$ & 1,00 & & & & \\
\hline $\mathrm{LGr}$ & $\begin{array}{c}0,02 \\
0,899 \\
\end{array}$ & $\begin{array}{l}-0,08 \\
0,405 \\
\end{array}$ & $\begin{array}{l}-0,04 \\
0,670 \\
\end{array}$ & $\begin{array}{c}0,07 \\
0,444 \\
\end{array}$ & $\begin{array}{c}0,12 \\
0,172 \\
\end{array}$ & $\begin{array}{l}-0,29 \\
0,001 \\
\end{array}$ & $\begin{array}{l}-0,22 \\
0,014 \\
\end{array}$ & 1,00 & & & \\
\hline $1 \mathrm{Gr}$ & $\begin{array}{l}-0,03 \\
0,767 \\
\end{array}$ & $\begin{array}{l}-0,31 \\
0,000 \\
\end{array}$ & $\begin{array}{l}-0,18 \\
0,056 \\
\end{array}$ & $\begin{array}{l}-0,15 \\
0,109 \\
\end{array}$ & $\begin{array}{c}0,13 \\
0,148 \\
\end{array}$ & $\begin{array}{c}0,08 \\
0,411 \\
\end{array}$ & $\begin{array}{c}0,22 \\
0,015 \\
\end{array}$ & $\begin{array}{c}0,53 \\
0,000 \\
\end{array}$ & 1,00 & & \\
\hline Pd100G & $\begin{array}{l}-0,31 \\
0,001 \\
\end{array}$ & $\begin{array}{l}-0,49 \\
0,000 \\
\end{array}$ & $\begin{array}{l}-0,44 \\
0,000 \\
\end{array}$ & $\begin{array}{l}-0,74 \\
0,000 \\
\end{array}$ & $\begin{array}{c}0,12 \\
0,178 \\
\end{array}$ & $\begin{array}{c}1,00 \\
0,000 \\
\end{array}$ & $\begin{array}{c}0,48 \\
0,000 \\
\end{array}$ & $\begin{array}{l}-0,28 \\
0,002 \\
\end{array}$ & $\begin{array}{c}0,09 \\
0,371 \\
\end{array}$ & 1,00 & \\
\hline Rend & $\begin{array}{l}-0,01 \\
0,903 \\
\end{array}$ & $\begin{array}{l}-0,24 \\
0,008 \\
\end{array}$ & $\begin{array}{l}-0,33 \\
0,000 \\
\end{array}$ & $\begin{array}{l}-0,43 \\
0,000 \\
\end{array}$ & $\begin{array}{c}0,06 \\
0,476 \\
\end{array}$ & $\begin{array}{c}0,64 \\
0,000\end{array}$ & $\begin{array}{c}0,68 \\
0,000 \\
\end{array}$ & $\begin{array}{l}-0,45 \\
0,000 \\
\end{array}$ & $\begin{array}{c}0,06 \\
0,554 \\
\end{array}$ & $\begin{array}{c}0,64 \\
0,000 \\
\end{array}$ & 1,00 \\
\hline
\end{tabular}

Tableau 4: Valeurs propres et contribution des caractères aux axes de l'analyse en composante principale.

\begin{tabular}{lccc}
\hline & Axe 1 & Axe 2 & Axe 3 \\
\hline Valeur propre & 5,87 & 2,15 & 1,59 \\
Variance totale \% & 36,72 & 13,49 & 9,95 \\
Cumul de la variance totale $\%$ & 36,72 & 50,21 & 60,16 \\
\hline \multicolumn{4}{c}{ Caractères définissant les axes et leurs valeurs propres } \\
\hline Tem & 0,29 & 0,04 & $-0,41$ \\
Tfl (j) & 0,61 & 0,34 & 0,11 \\
NBr & 0,58 & 0,07 & 0,29 \\
Tma & 0,80 & 0,10 & 0,04 \\
NFp & $-0,33$ & $-0,19$ & $-0,49$ \\
NGs & $-0,85$ & 0,19 & 0,02 \\
LGS & $-0,69$ & 0,02 & 0,24 \\
LGr & 0,24 & $-0,76$ & $-0,29$ \\
lGr & $-0,24$ & $-0,76$ & $-0,13$ \\
Pd100G & $-0,85$ & 0,19 & 0,02 \\
Rend & $-0,74$ & 0,31 & 0,23 \\
\hline
\end{tabular}


A. A. GBAGUIDI et al. /Int. J. Biol. Chem. Sci. 9(2): 1050-1066, 2015

Tableau 5: Quelques caractéristiques agro morphologiques des variétés élites des morphotypes.

\begin{tabular}{|c|c|c|c|c|c|}
\hline Noms vernaculaires & Longueur gousse (cm) & Nombre de gousse/plant & Cycle (JAS) & Poids (g) 100 graines & Rendement (Kg/ha) \\
\hline Tiforga (N73) & $12 \pm 5,7$ & $14,67 \pm 0,5$ & $75 \pm 0,0$ & $14 \pm 9,1$ & $517,6 \pm 5,01$ \\
\hline Touncalaou (N74) & $12 \pm 2,3$ & $14,33 \pm 2,0$ & $92 \pm 1,5$ & $14,68 \pm 3,2$ & $518,7 \pm 3,8$ \\
\hline Dougourikouaré (N35) & $17,33 \pm 1,4$ & $16 \pm 8,0$ & $95 \pm 0,0$ & $15,54 \pm 5,3$ & $674,3 \pm 2,5$ \\
\hline Tola (N56) & $14,67 \pm 0,7$ & $17 \pm 6,5$ & $90 \pm 2,3$ & $15,91 \pm 4,8$ & $730 \pm 4,01$ \\
\hline Malanville sui (N59) & $14,67 \pm 3,1$ & $17 \pm 1,5$ & $93 \pm 0,5$ & $15,91 \pm 2,5$ & $730,3 \pm 0,2$ \\
\hline Tounbopessi (N48) & $15,83 \pm 3,5$ & $57,33 \pm$ & $52 \pm 0,3$ & $19,33 \pm 0,0$ & $885 \pm 5,7$ \\
\hline Wlétchivé (N12) & $15,33 \pm 4,2$ & $32 \pm 0,0$ & $72 \pm 0,0$ & $10,96 \pm 0,4$ & $929,7 \pm 9,01$ \\
\hline Sakaoga (N121) & $21,33 \pm 0,2$ & $20,67 \pm 15,5$ & $50 \pm 0,5$ & $17,13 \pm 6,7$ & $984,5 \pm 3,2$ \\
\hline TinforgaTapoga (N70) & $15 \pm 1,0$ & $14,33 \pm 9,8$ & $93 \pm 0,1$ & $14,93 \pm 3,3$ & $997,6 \pm 4,4$ \\
\hline Tihiman (N60) & $14,67 \pm 3,3$ & $17 \pm 6,5$ & $52 \pm 0,1$ & $17,57 \pm 0,5$ & $1120,5 \pm 0,32$ \\
\hline Kakéé (N66) & $16,67 \pm 4,5$ & $61,67 \pm 49,5$ & $93 \pm 0,4$ & $14,84 \pm 2,7$ & $1129,4 \pm 1,7$ \\
\hline Kpodjiguèguè (N91) & $13,67 \pm 2,5$ & $48,67 \pm 3,0$ & $65 \pm 0,2$ & $22,99 \pm 0,0$ & $1264,5 \pm 2,4$ \\
\hline Ejè (N108) & $17,33 \pm 2,6$ & $26 \pm 9,0$ & $75 \pm 0,1$ & $18,06 \pm 6$ & $1276,1 \pm 5,2$ \\
\hline Safoto (N95) & $16,83 \pm 0,0$ & $37 \pm 7,5$ & $51 \pm 0,01$ & $23,75 \pm 1,5$ & $1378,3 \pm 0,5$ \\
\hline Egbanmonlou (N68) & $18 \pm 5$ & $44 \pm 13,8$ & $52 \pm 0,2$ & $20,74 \pm 2,4$ & $1570,3 \pm 6,1$ \\
\hline Kplobè (N112) & $17 \pm 3,4$ & $48,67 \pm 20,2$ & $54 \pm 0,8$ & $16,45 \pm \quad 0,04$ & $1679,4 \pm 0,9$ \\
\hline Assoban'dé (N36) & $17,33 \pm 9$ & $47,33 \pm 27,0$ & $52 \pm 0,1$ & $15,93 \pm 1,1$ & $1685 \pm 0,00$ \\
\hline Wandamin (N39) & $17,33 \pm 4,1$ & $58 \pm 2,0$ & $89 \pm 0,5$ & $12,82 \pm 7,3$ & $1696 \pm 2,9$ \\
\hline Samahangui (N77) & $17,83 \pm 2,3$ & $25,33 \pm 2,5$ & $93 \pm 0,03$ & $19,12 \pm 3,2$ & $1807,1 \pm 3,12$ \\
\hline Yanbodo (N102) & $21,33 \pm 3,2$ & $53,33 \pm 0,0$ & $51 \pm 0,07$ & $18,34 \pm 0,7$ & $2459 \pm 1,8$ \\
\hline Gbolékpomin (N101) & $18,67 \pm 2,1$ & $33,33 \pm 19,7$ & $91 \pm 0,0$ & $14,95 \pm 4,1$ & $2587,5 \pm 8,3$ \\
\hline Catché godonou (N82) & $16,33 \pm 3,3$ & $40 \pm 10,4$ & $52 \pm 0,9$ & $18,01 \pm 2,5$ & $2617,3 \pm 1,1$ \\
\hline Kaki (N113) & $17,33 \pm 4,5$ & $70,66 \pm 6,8$ & $74 \pm 0,1$ & $20,93 \pm 8,0$ & $2684,2 \pm 2,7$ \\
\hline Ewaoloy (N120) & $22 \pm 1$ & $64,33 \pm 18,3$ & $93 \pm 1,3$ & $23,75 \pm 0,4$ & $2696,5 \pm 11$ \\
\hline
\end{tabular}



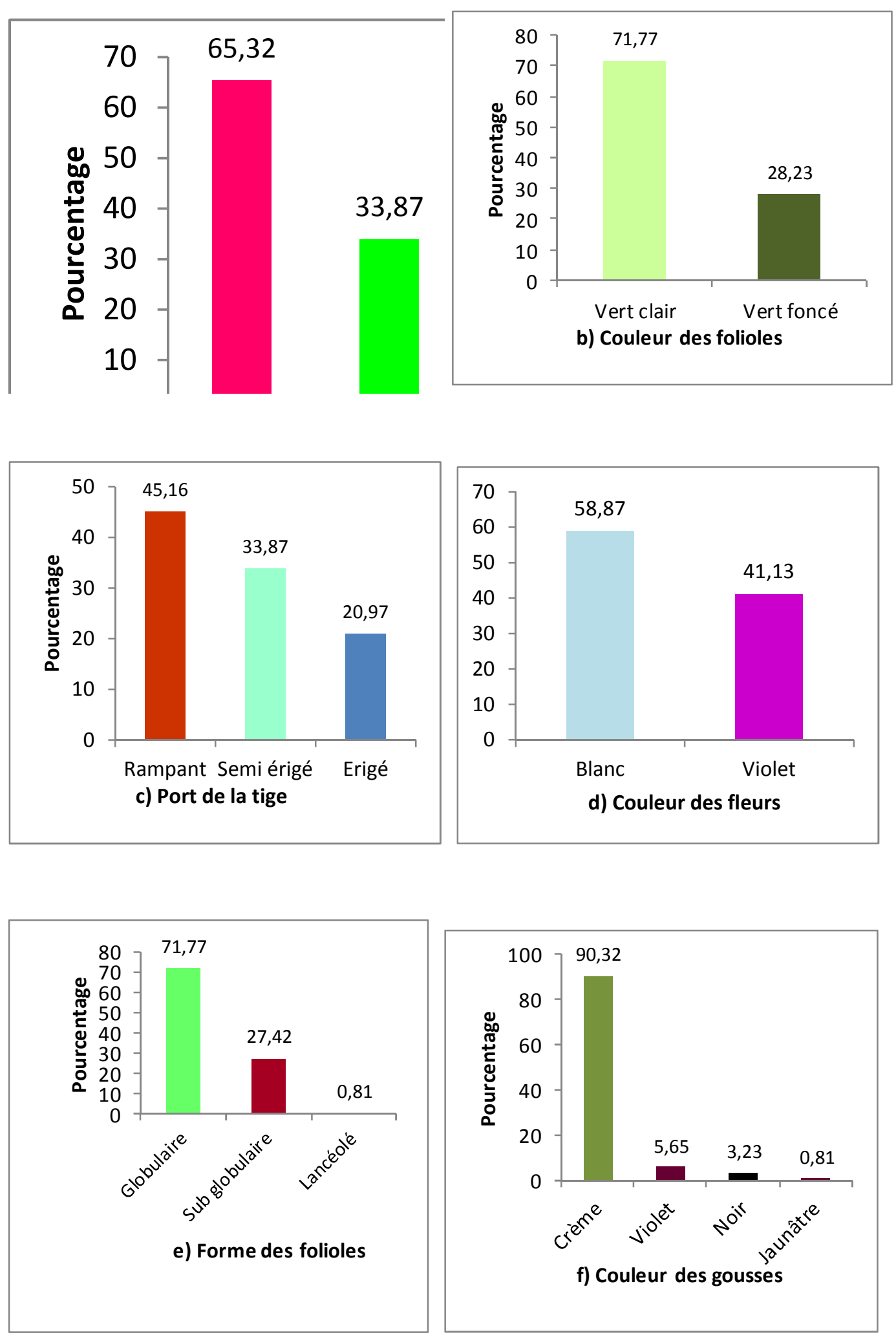

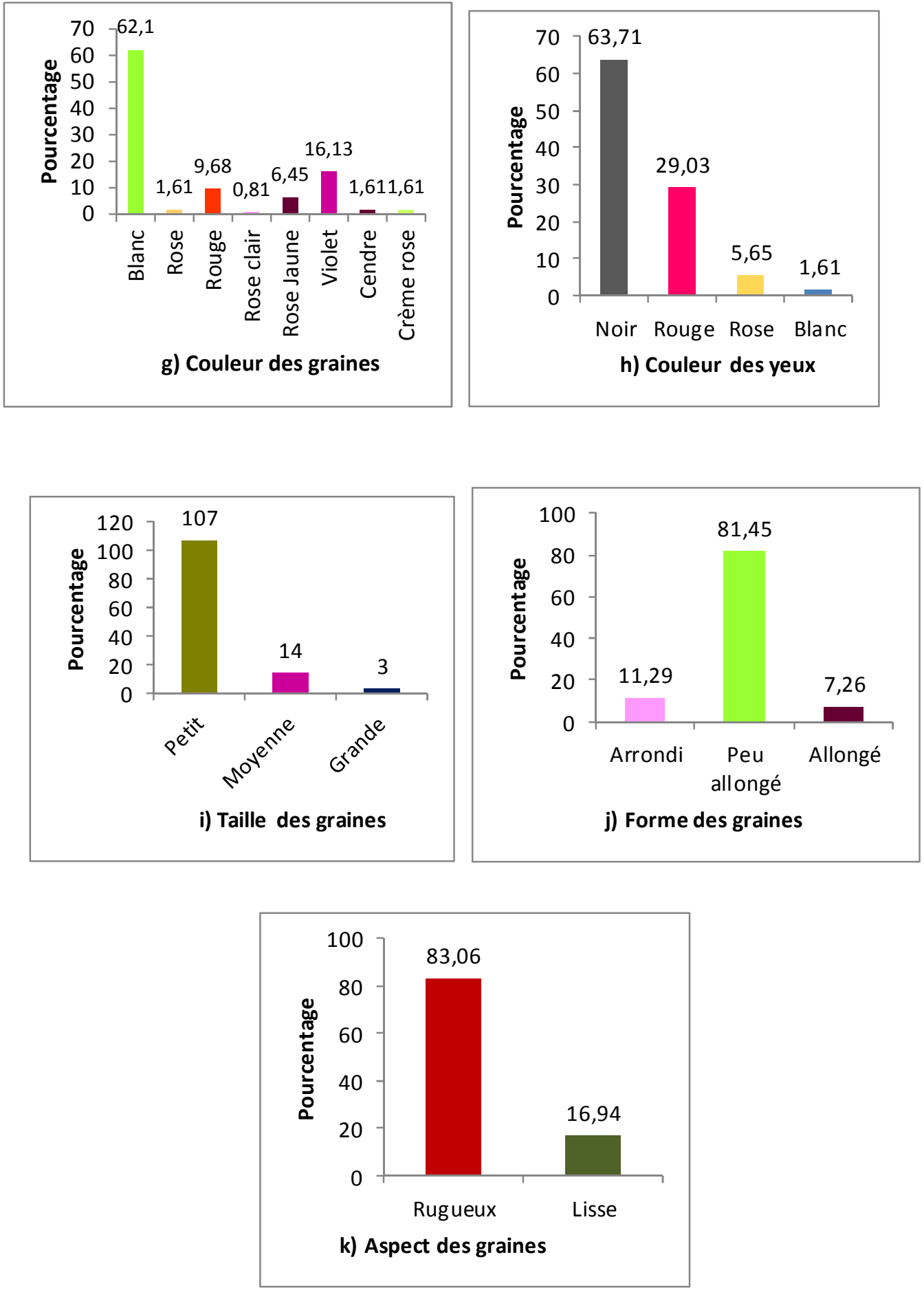

Figure 1 : Fréquence des variables qualitatives au sein des variétés de niébé collectées au Bénin. 


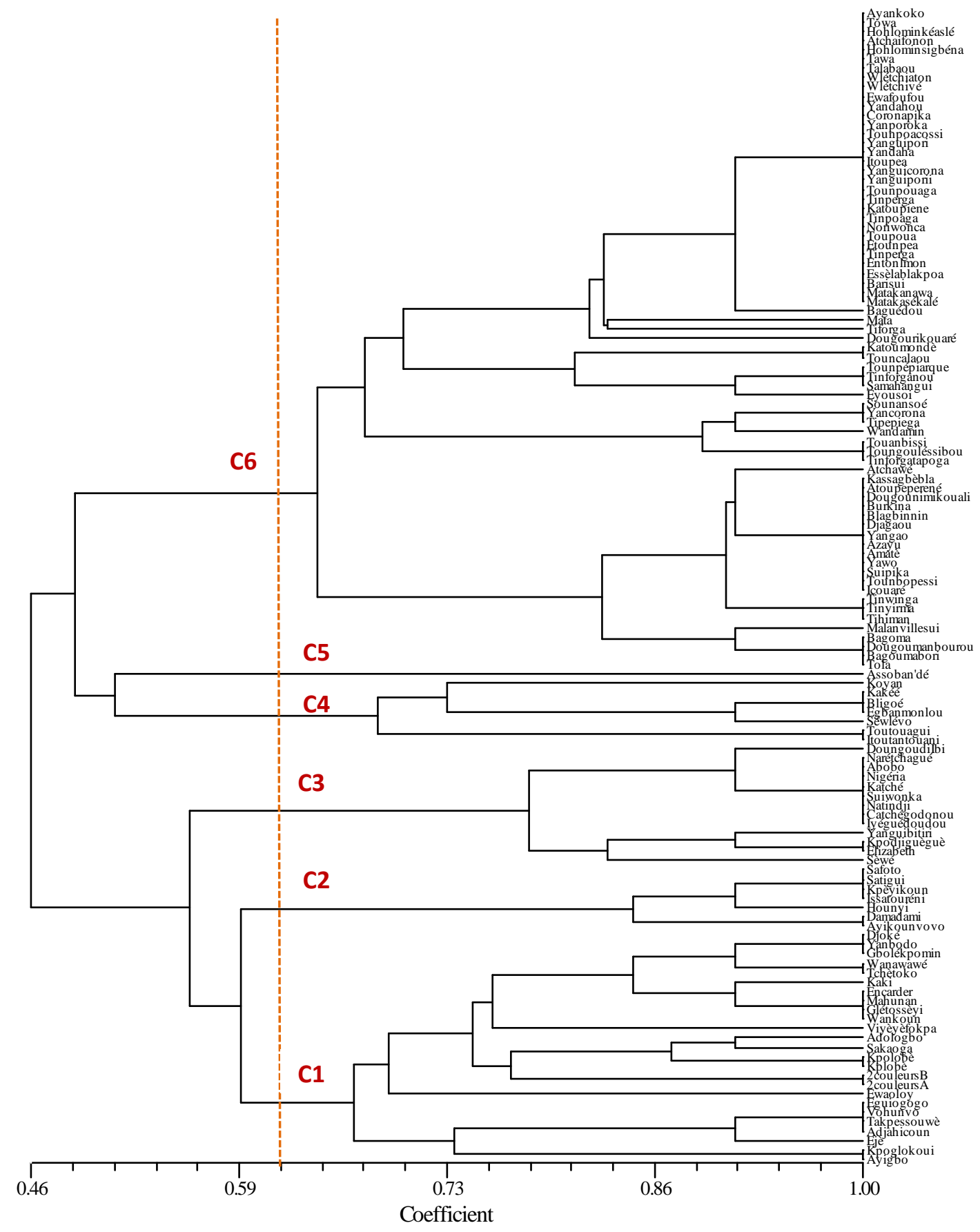

Figure 2 : Classification ascendante hiérarchique des variétés collectées. 


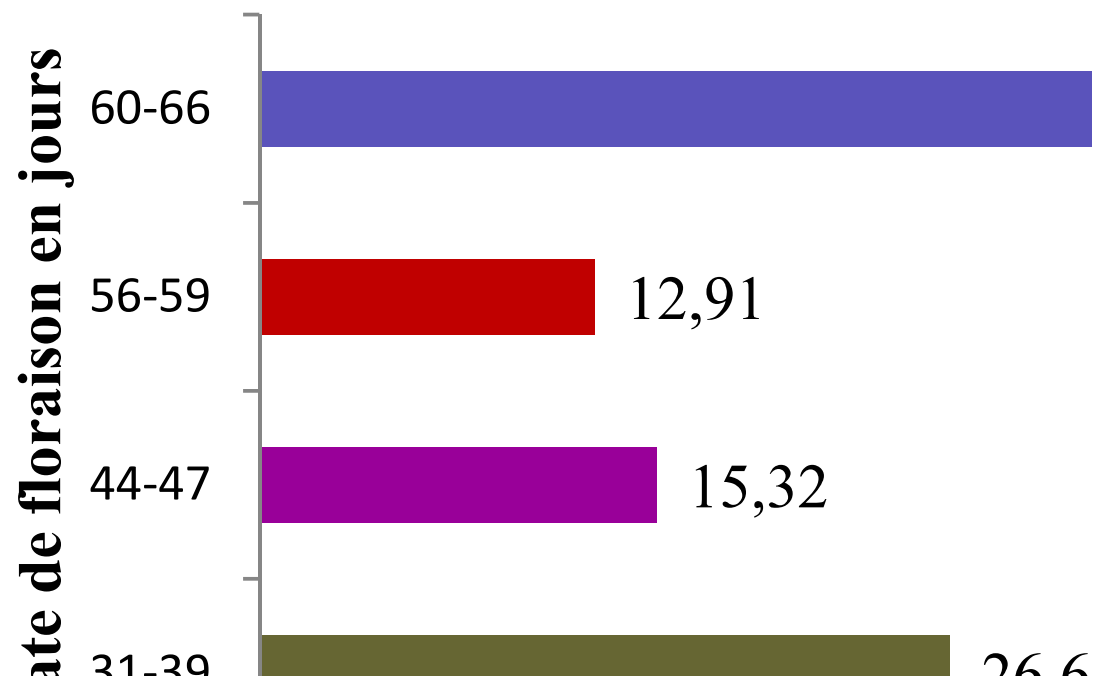

Figure 3: Date en jours de 50\% de floraison.

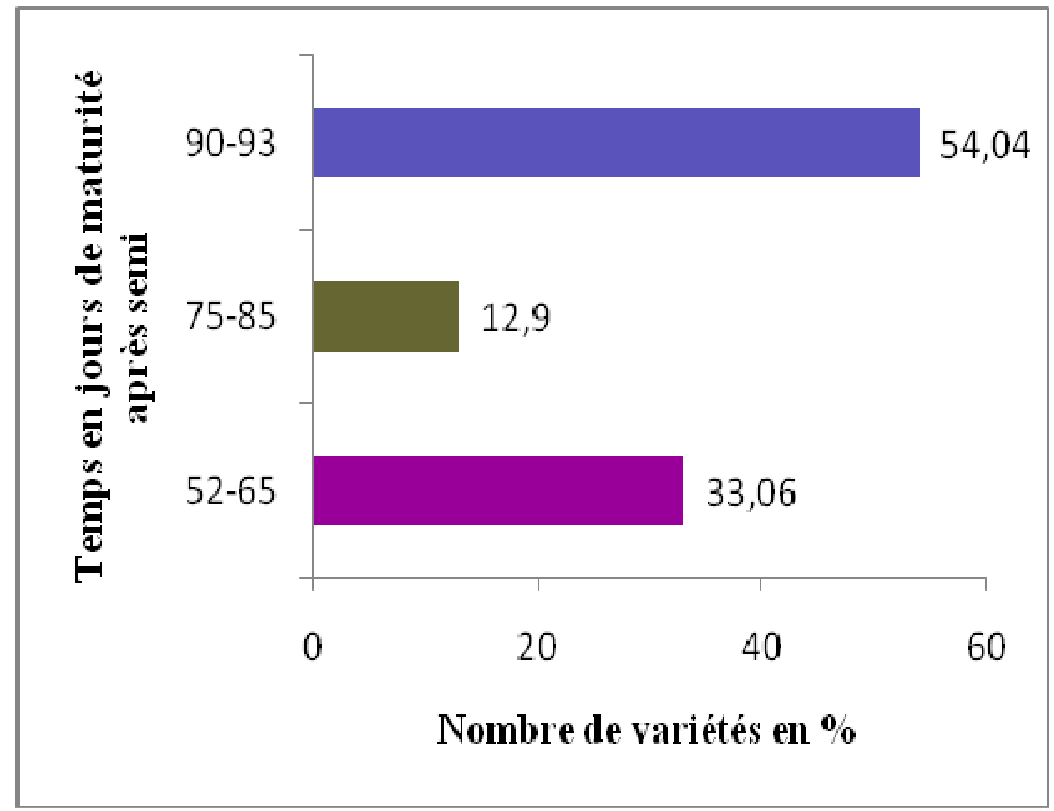

Figure 4: Cycle des variétés en jours. 
Projection desind. sur le plan factoriel $\left(\begin{array}{ll}1 \times 2 & 2\end{array}\right)$

Observations avec la somme des cosinus carrés $>=0,00$

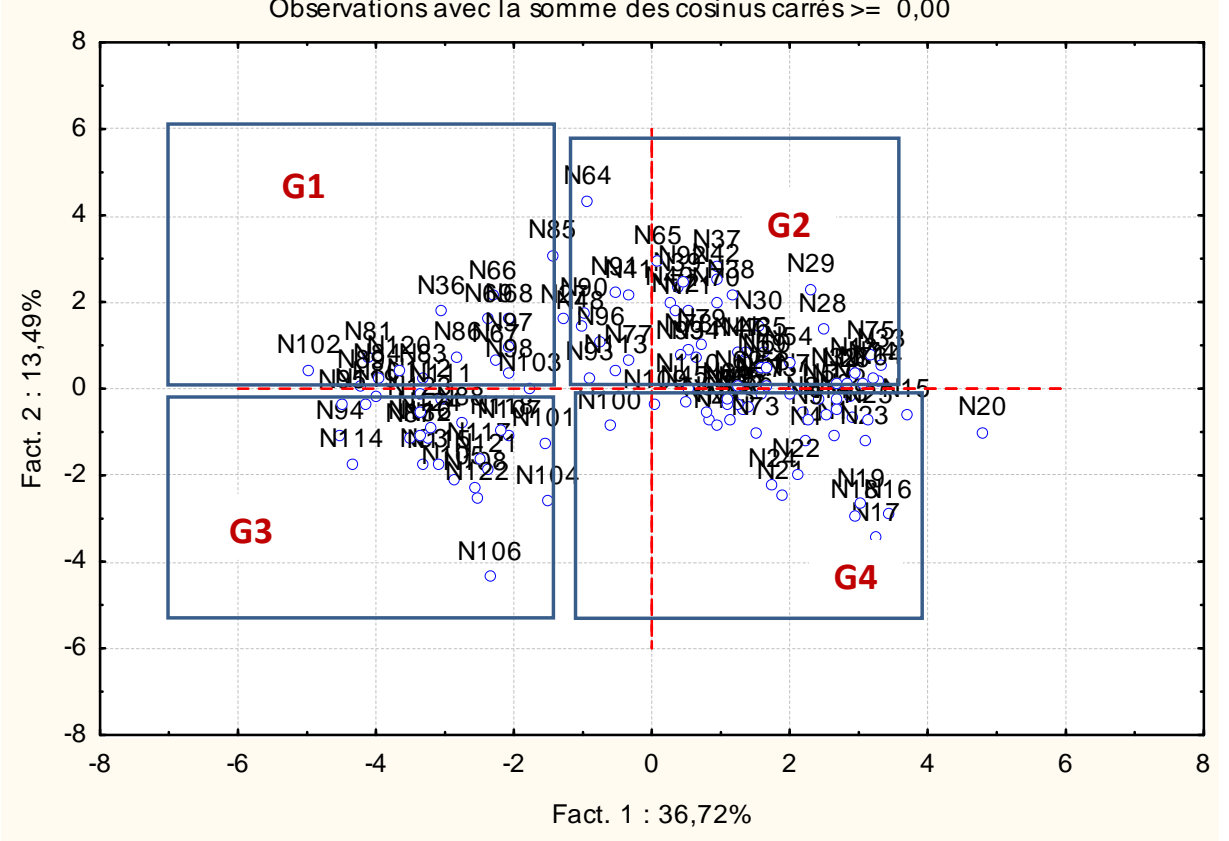

Figure 5 : Projection des 124 variétés dans le plan factoriel 1 et 2.

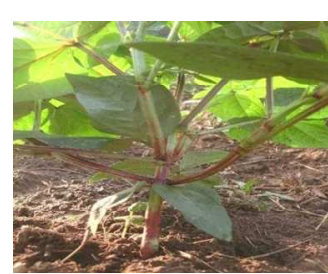

a1

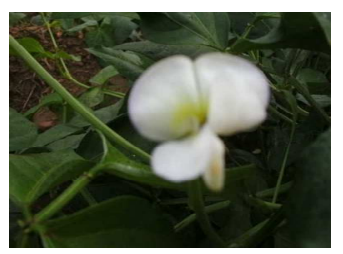

a2

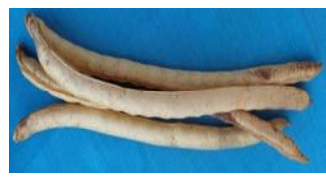

a3

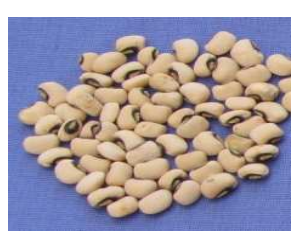

a4

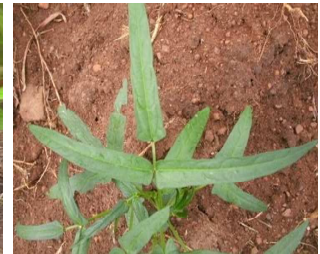

b1

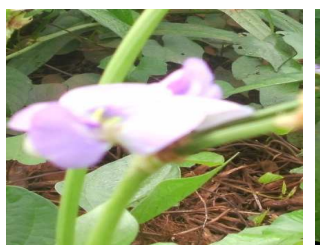

b2

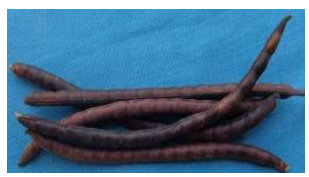

b3

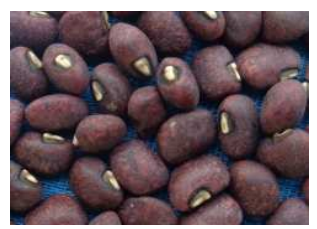

b4

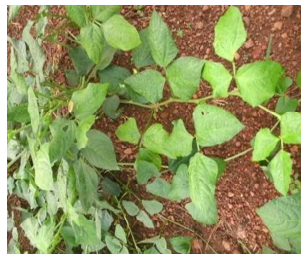

c1

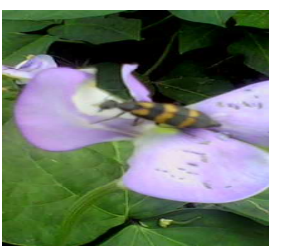

$\mathrm{c} 2$

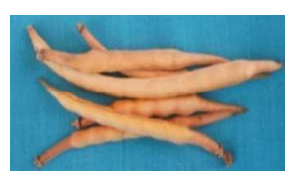

c3

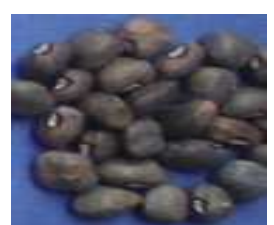

$\mathrm{c} 4$

Figure 6 : Caractéristiques de quelques morphotypes.

a1 (plante), a2 (fleur), a3 (gousse) et a4 (graines) de la variété Wlétchivé ; b1 (Plante), b2 (fleur), b3 (gousse) et b4 graines de la variété Abobo; c1 (Plante), c2 (Fleur), c3 (gousse), c4 (graines) de la variété Kpodjiguèguè 


\section{DISCUSSION}

L'utilisation rationnelle de la diversité génétique du niébé au Bénin exige une bonne connaissance des caractéristiques des variétés. La présente étude a permis d'apprécier le statut actuel de la diversité de niébé d'une part et d'identifier les variétés performantes d'autres part. L'analyse des caractères qualitatifs tels que la pigmentation de la plante, la couleur et la forme des folioles, la couleur des fleurs, le port de la tige, les caractéristiques des gousses et des graines a montré une grande variabilité au sein de la collection étudiée. Des résultats similaires ont été obtenus par Stoilova et Pereira (2013). En plus des couleurs blanche et violette des fleurs retrouvées dans notre étude, Cobbinah et al. (2011) ont réalisé dans des travaux similaires des fleurs de couleur blanc-violette et ont trouvé un pourcentage plus élevé de variétés de fleurs violettes. La classification ascendante hiérarchique faite à partir de ces variables qualitatives, regroupe les 124 variétés en 42 unités morphologiques. Ce qui suppose l'existence de doublons au sein de la collection étudiée. Cette situation pourrait être expliquée par le fait qu'au sein d'une collection, une même variété peut avoir plusieurs noms comme ce fut le cas chez l'igname (Loko et al., 2013) et le fonio (Dansi et al., 2010). Cette caractérisation a donc permis d'identifier ces doublons et d'établir une équivalence entre les variétés collectées comme l'ont fait Kuruma et al. (2008) au Kénya pour le niébé et Basu et al. (2007a et b) au Botswana sur le voandzou.

Les moyennes obtenues pour chaque caractère quantitatif sont nettement supérieures à celles observées dans des études similaires réalisées par Cobbinah et al. (2011) au Ghana, Makanur et al. (2013) en Inde mais elles sont plus faibles que celles obtenues par Ouédraogo et al. (2010) au Burkina Faso sur la même espèce. Les coefficients de variation élevés observés pour un nombre significatif de caractères indiquent la présence d'une forte hétérogénéité au sein des variétés collectées. Les résultats pourraient être aussi en partie le reflet de la sensibilité du niébé aux variations de la photopériode (Andargie et al., 2013). En effet, de nombreux travaux ont montré que la longueur du jour entraîne des effets variables sur le développement végétatif et physiologique du niébé (Mukhtar et Singh, 2006 ; Gonné et al., 2013 ; Nuhu et Mukhtar, 2013). Le même constat est fait par Bonny et Djè (2011) sur le voandzou en Côte d'ivoire. La moyenne de 52 JAS enregistrée pour la floraison des variétés traditionnelles du Bénin étudiées est similaire aux résultats obtenus en Inde par Makanur et al. (2013) avec 35 accessions et largement plus élevée que ceux obtenus par Cobbinah et al. (2011) qui ont fait une étude similaire sur 136 accessions au Ghana. Des variétés très précoces (53 JAS) ont été aussi identifiées par Doumbia et al. (2013) dans 94 accessions du Ghana et du Mali. La précocité des variétés de niébé est une caractéristique agronomique importante qui pourrait contribuer à faire face aux phénomènes des changements climatiques et particulièrement à la sècheresse. Cette précocité peut être évaluée par le temps de floraison ou de maturation des gousses (Cobbinah et al., 2011). Selon les mêmes auteurs, certaines variétés peuvent fleurir 30 jours après semis pour murir 25 jours plus tard. La variété kpodjiguèguè qui est précoce n'a malheureusement pas une forte valeur marchande à cause de la couleur violette tachetée de brun sur le tégument de la graine. Ceci confirme les résultats obtenus par Zannou et al. (2013) qui ont démontré que les prix varient selon les variétés de niébé et que le marché valorise différemment leurs traits distinctifs. Selon Shanko et al. (2014) le temps de floraison, le temps de maturation et le nombre de gousses par plante ont des effets négatifs sur le rendement du niébé. Tout programme d'amélioration variétale étant basé sur la diversité génétique (Stoilova et Pereira, 2013), les classes obtenues à partir des caractères quantitatifs donnent une idée de la performance des variétés de niébé cultivées au Bénin. De bonnes lignées parentales pourront être obtenues des groupes 1 et 3 en vue d'avoir des descendants à graines de grandes tailles et à haut rendement. De plus, les 
variétés de grande taille sont intéressantes pour les consommateurs et présentent souvent de bonnes qualités technologiques (Doumbia et al., 2013). Ce sont des caractéristiques que possèdent les variétés Ewaoloy, Kaki et Yanbodo qui peuvent être utilisées par les sélectionneurs dans les programmes d'amélioration variétale. Cette différence de poids de 100 graines et de rendements observée au niveau des variétés de niébé au Bénin a été déjà évoquée dans une étude similaire faite par Doumbia et al. (2013) sur des accessions de niébé du Ghana et du Mali et qui estiment que l'accumulation des réserves dans les graines dépend du type de génotype mais également des facteurs climatiques (Khan et al., 2010).

Pour le maintien de la diversité, des stratégies de conservation in situ et ex situ de ces variétés sont nécessaires. Catché godonou, Ewaoloy, Gbolékpomin, Kaki et Yanbodo sont des variétés à haut rendement de plus de 2 tonnes à l'hectare. En plus de la forte productivité, les variétés Catché godonou et Kaki sont très précoces avec respectivement des cycles de 52 et 74 jours après semis (Tableau 5). Ces variétés sont à promouvoir dans le contexte actuel des changements climatiques. La variété kaki de couleur violette serait plus appréciée par les consommateurs, contrairement à la variété Catché godonou de couleur noirâtre. Pour tenir compte de ce critère, la variété kaki de couleur violette est un bon candidat pour des programmes de production de semences certifiées et d'amélioration variétale. Néanmoins, une caractérisation moléculaire des variétés collectées est nécessaire et, permettra d'éliminer les doublons. Cette démarche a été déjà adoptée par Ghalmi et al. (2010) en Algérie, Kuruma et al. (2008) au Kenya et Ouédraogo et al. (2010) au Burkina Faso.

\section{Conclusion}

L'ensemble de ces résultats témoigne de l'existence d'une diversité importante au sein de la collection de niébé au Bénin. Cette importante variabilité phénotypique observée pourrait résulter de l'expression d'une forte hétérogénéité génotypique mais aussi de l'influence des facteurs environnementaux. Les tests de régression linéaire ont indiqué une corrélation significative entre les caractères morphologiques et le rendement des variétés. Les variétés à cycle court qui présentent de bon rendement comme Kaki et Catché Godonou sont de bons candidats pour les programmes de sélection variétale. Une caractérisation moléculaire est nécessaire pour clarifier les synonymies et pour une utilisation rationnelle des ressources disponibles.

\section{REMERCIEMENTS}

Nous exprimons notre profonde reconnaissance à l'Etat béninois pour la bourse de thèse qui nous a été accordée, aux Docteurs Arlette ADJATIN, Laura LOKO et Eben-Ezer pour les corrections apportées à cet article. Nous témoignons toutes nos reconnaissances à tous nos collègues en particulier Angelot AGRE, Innocent DOSSOU-AMINON et Azize OROBIYI pour leur assistance technique et soutien de tous ordres. A tous les chefs des villages visités au cours des prospections, nous disons merci pour l'accueil chaleureux qu'ils nous ont réservé.

\section{REFERENCES}

Abadassi J. 2014. Agronomic Traits of Cowpea (Vigna unguiculata (L.) Walp.) Populations cultivated in Benin. International Journal of Science and Advanced Technology, 4(2): 4.

Abebe G, Assefa T, Harrun H, Mesfine T, AlTawahaA-RM. 2005. Participatory selection of drought tolerant maize varieties using mother and baby methodology: a case study in the semiarid zones of the Central Rift Valley of Ethiopia. World J Agric Sci., 1(1): 22-27.

Adjatin A, Dansi A, Eze CS, Assogba P, Dossou-Aminon I, Akpagana K, Akoégninou A, Sanni A. 2012. Ethnobotanical investigation and diversity of Gbolo (Crassocephalum rubens (Juss. ex Jacq.) S. Moore and Crassocephalum crepidioides (Benth.) $\mathrm{S}$. Moore), a traditional leafy vegetable 
under domestication in Benin. Genet. Resour. Crop Evol., 59(8):1867-1881.

Akoégninou A, van der Burg WJ, van der Maesen. 2006. Flore Analytique de Bénin. Backhuys Publishers: Leiden; 1034.

Andargie M, Pasquet RS, Muluvi GM, Timko MP. 2013. Quantitative trait loci analysis of flowering time related traits identified in recombinant inbred lines of cowpea (Vigna unguiculata); Genome, 56: 289294.

Basu S, Mayes S, Davey M, Jeremy A, Roberts Sayed N, Azam-Ali Richard Mithen, Remy S. Pasquet. 2007a. Inheritance of 'domestication' traits in Bambara groundnut (Vigna subterranea (L.) Verdc). Euphytica, 157: 59-68.

Basu S, Roberts JAS, Azam-Ali N, Mayes. 2007b. Development of microsatellite markers for Bambara groundnut (Vigna subterranea L. Verdc.) - an underutilized African legume crop species. Molecular Ecology Notes, 7: 1326-1328.

Bonny BS, Djè Y. 2011. Variabilité morphologique et agronomique des variétés traditionnelles de voandzou [Vigna subterranea (L.) Verdc. (Fabaceae)] de Côte d'Ivoire. Journal of Applied Bioscience, 41: 2820-2835.

Carsky RJ, Vanlauwe B, Lyasse O. 2002. Cowpea Rotation as a Resource Management Technology for CerealBased Systems in the Savannas of West Africa. In Fatokun CA, Tarawali SA, Singh BB, Kormawa PM, Tamo M (eds) Challenges and Opportunities for Enhancing Sustainable Cowpea Production. International Institute of Tropical Agriculture: Ibadan, Nigeria; 252-266.

Cobbinah FA, Addo-Quaye AA, Asante IK. 2011. Characterization, Evaluation And Selection Of Cowpea (Vigna unguiculata (L.) Walp) accessions with desirable traits from eight regions of ghana, ARPN Journal of Agricultural and Biological Science, 6(7): 21-32.

Coulibaly S, Pasquet RS, Papa R, Gepts P. 2002. AFLP analysis of the phenetic organization and genetic diversity of cowpea [Vigna unguiculata (L.) Walp.] reveals extensive gene flow between wild and domesticated types. Theor. Appl. Genet., 104: 258-266.

Dansi A, Adoukonou-Sagbadja H, Vodouhé R. 2010. Diversity, conservation and related wild species of Fonio millet (Digitaria spp.) in the northwest of Benin. Genet. Resour. Crop Evol., 57: 827-839.

Doumbia IZ, Akromah R, Asibuo JY. 2013. Comparative study of cowpea germplasms diversity from Ghana and Mali using morphological characteristics. J. Plant Breed. Genet., 01(03) 139-147.

FAOSTAT. 2010. Agricultural production, crop primary database. Food and Agricultural Organization of the United Nations, Rome. http://faostat.fao.org/ faostat

Gbaguidi AA, Dansi A, Loko LY, Dansi M, Sanni A. 2013. Diversity and agronomic performances of the cowpea (Vigna unguiculata Walp.) landraces in Southern Benin. International Research Journal of Agricultural Science and Soil Science, 3(4): 121-133.

Ghalmi N, Malice M, Jacquemin JM, Ounane SM, Mekliche L, Baudoin JP. 2010. Morphological and molecular diversity within Algerian cowpea (Vigna unguiculata L. Walp. landraces. Genet. Resour. Crop Evol., 57: 371-386.

Gonné S, Wirnkar LV, Laminou A. 2013. Characterization of Some Traditional Cowpea Varieties Grown by Farmers in the Soudano-Sahelian Zone of Cameroon. International Journal of Agriculture and Forestry, 3(4): 170-177.

Hedge SV, Mishra KS. 2009. Landraces of cowpea, Vigna unguiculata (L.) Walp. As potential sources of genes for unique characters in breeding. Genetic Resources Crop Evolut., 56: 615-627.

Houinsou FRL, Adjou SE, Ahoussi ED, Sohounhloué CKD, Soumanou MM. 2014. Bioactivity of essential oil from fresh leaves of Lantana camara against fungi isolated from stored cowpea in 
southern Benin. International Journal of Biosciences, 5(1): 365-372.

IBPGR. 1983. Descriptors for Cowpea. IBPGR: Rome.

Khan A, Bari A, Khan S, Shan NH, Zada I. 2010. Performance of cowpea genotypes at higher altitude of NWFP. Pak. J. Bot., 42(4): 2291-2296.

Kombo GR, Dansi A, Loko LY, Orkwor GC, Vodouhe R, Assogba P, Magema JM. 2012. Diversity of cassava (Manihot esculenta Crantz) cultivars and its management in the department of Bouenza in the Republic of Congo. Genetic Resources and Crop Evolution, 59(8):1789-1803.

Kuruma RW, Kiplagat O, Ateka E, Owuoche G. 2008. Genetic diversity of Kenyan cowpea accessions based on morphological and microsatellite markers. East Afr. Agric. For. J., 76: 3-4.

Loko YL, Dansi A, Linsoussi C, Tamo M, Vodouhè R, Akoegninou A, Sanni A. 2013. Current status and spatial analysis of Guinea yam Dioscorea cayenensis Lam. -D. rotundata Poir. complex) diversity in Benin. International Research Journal of Agricultural Science and Soil Science, 3(7): 219-238.

Makanur B, Deshpande VK, Vyakaranahal BS. 2013. Characterisation of cowpea genotypes based on quantitative descriptors. Academic Journals, 8(4): 1183-1188.

MAEP (Ministère de l'Agriculture de l'Elevage et de la Pêche). 2012. Données statistiques des spéculations au Bénin, MAEP.

Mukhtar FB, Singh BB. 2006. Influence of photoperiod and Gibberellic acid (GA3) on the growth and flowering of Cowpea (Vigna unguiculata L. Walp). Journal of food, Agriculture and Environment, 4(2): 201-203.

Niba SA. 2011. Arthropod assemblage dynamics on cowpea (Vigna unguiculata L. Walp) in a subtropical agro-ecosystem, South Affric. African Journal Research, 6(4): 1009-1015.
Nkouannessi M. 2005. The genetic, morphological and physiological evaluation of African cowpea genotypes. Thesis, University of the Free State Bloemfontein, South Africa, 131pp.

Nuhu Y, Mukhtar FB. 2013. Screening of some cowpea genotypes for photosensitivity. Bayero Journal of Pure and Applied Sciences, 6(2): 31 - 34.

Rohlf FJ. 2009. NTSYSpc: Numerical Taxonomy System. ver. 2.21c. Exeter Software. Setauket: New York.

Sanginga N, Dashiell KE, Diels J, Vanlauwe B, Lyasse O, Carsky RJ, Tarawali S, Asafo-Adjei B, Menkir A, Schulz S, Singh BB, Chikoye D, Keatinge D, Ortiz R. 2003. Sustainable resource management coupled to resilient germplasm to provide new intensive cereal -grain-legume livestock systems in the dry savanna. Agric. Ecosyst. Environ., 100: 305-314.

Shanko D, Andargie M, Zelleke H. 2014. Interrelationship and Path Coefficient Analysis of Some Growth and Yield Characteristics in Cowpea (Vigna unguiculata L. Walp) Genotypes. Journal of Plant Sciences. 2(2): 97-101.

Sneath PHA, Sokal RO. 1973. Numerical Taxonomy. Freeman: San Francisco.

Stoilova T, Pereira G. 2013. Assessment of the genetic diversity in a germplasm Collection of cowpea (Vigna unguiculata (L.) Walp.) using morphological traits. African Journal of Agricultural Research, 8(2): 208-215.

Swofford DL, Olsen GJ. 1990. Phylogeny reconstruction. In Molecular Systematic, Hillis DM, Moritz C (eds). Sinauer Associates: Sunderland, Mass.

Ouedraogo JT, Sawadogo M, Tignegre JB, Drabo I, Balma D. 2010. Caractérisation agromorphologique et moléculaire de cultivars locaux de niébé (Vigna unguiculata) du Burkina Faso. Cameroon Journal of Experimental Biology, 06(1) 31-40.

Ouali-N'goran SWM, Boga JP, Johnson F, Tano Y, Fouabi K. 2014. Influence of dietary factors of five varieties of beans 
sold in Côte d'Ivoire on some biological parameters of Callosobruchus maculatus (Fab.) Coleoptera, Bruchidae. Journal of Animal \& Plant Sciences, 21(1): 32513262.

Taffouo VD, Ndongo Din JE, Nguelemeni MP, Eyambé YM, Tayou RF, Akoa A. 2008. Effets de la densité de semis sur la croissance, le rendement et les teneurs en composés organiques chez cinq variétés de niébé (Vigna unguiculata L. Walp). Journal of Applied Biosciences, 12: 623 632.

Tarawali SA, Singh BB, Gupta SC, Tabo R, Harris F, Nokoe S, Fernández-Rivera S, Bationo A, Manyong VM, Makinde K, Odion EC. 2002. Cowpea as a key factor for a new approach to integrated croplivestock systems research in the dry savannas of West Africa. In Challenges and Opportunities for Enhancing Sustainable Cowpea Production, Fatokun CA, Tarawali SA, Singh BB, Kormawa PM, Tamo M (eds). International Institute of Tropical Agriculture: Ibadan, Nigeria; 233-251.

Zannou A, Ahanchédé A, Struik PC, Richards P, Zoundjihékpon J, Tossou R, Goodhue S, 2004. Yam and cowpea diversity management by farmers in the guineasudan transition zone of Benin. NJAS Wageningen Journal of Life Sciences, 52(3-4): 393-420.

Zannou A, Struik PC, Richards P. 2013. The value of cowpea (Vigna unguiculata) Diversity in the transition Guinea Sudan Zone of Benin: Market evidence. Annales des Sciences Agronomiques, 17(2): 183196. 